Voix et Images

voixetimages

\title{
Renaissance du roman social?
}

\section{Jacques Pelletier}

Volume 8, numéro 2, hiver 1983

Marie-Claire Blais

URI : https://id.erudit.org/iderudit/200392ar

DOI : https://doi.org/10.7202/200392ar

Aller au sommaire du numéro

\section{Éditeur(s)}

Les Presses de l’Université du Québec

\section{ISSN}

0318-9201 (imprimé)

1705-933X (numérique)

Découvrir la revue

\section{Citer cet article}

Pelletier, J. (1983). Renaissance du roman social? Voix et Images, 8(2), 371-377. https://doi.org/10.7202/200392ar d'utilisation que vous pouvez consulter en ligne.

https://apropos.erudit.org/fr/usagers/politique-dutilisation/ 
ROMAN

\title{
Renaissance du roman social?
}

\author{
par Jacques Pelletier, Université du Québec à Montréal
}

Comme tout le monde j'aime lire pour le plaisir du texte comme disait l'autre: après tout, la littérature constitue une distraction, un moyen d'évasion - elle possède une incontestable dimension ludique: et pourquoi pas? mais elle se présente aussi comme un mode privilégié d'approche de la réalité et, l'analysant, ce qui me passionne d'abord en elle, ce sont justement les rapports qu'entretiennent l'imaginaire et le réel. $\dot{A}$ ce titre, les romans qui font l'objet de cette chronique sont particulièrement intéressants en ce qu'ils sont inspirés, de manière explicite ou implicite, par cette problématique qui se profile également derrière de nombreuses productions depuis un an ou deux si bien qu'on peut se demander si on n'assiste pas à une renaissance du roman social après une décennie dominée par diverses variantes du formalisme né de la conjoncture culturelle et littéraire au début des années 1970 ?

Gilles Raymond, "compagnon de route" sinon militant des organisations marxistes-léninistes à la belle époque où elles connaissaient une ascension fulgurante, a publié jusqu'ici deux romans qu'on ne peut qualifier autrement que d'engagés au sens le plus fort du terme. En 1979, la troupe de théâtre - Les gens d'en bas` devenait, pour l'occasion, éditeur de son premier roman au titre évocateur: Pour sortir de nos cages ${ }^{1}$, titre qui constituait à lui seul tout un programme, le «pour" indiquant déjà nettement la volonté didactique de l'auteur qui s'identifiait à des personnages "en cages», à délivrer donc.

Dans son Avant-propos, Raymond affirmait effectivement on ne peut plus clairement: «avec ses personnages, ses situations, son action, l'écriture de ce roman affirme en elle-même le mouvement de la classe ouvrière québécoise et canadienne vers son émancipation* (p. 5) et invitait ses lecteurs, si la lecture de son roman les avait convaincus, à "le faire circuler parmi vos compagnons de travail, vos amis» (p. 6).

Ce propos militant, l'auteur choisissait, pour l'illustrer, la mise en ścène d'une grève dans une entreprise moyenne comptant trois cent travailleurs, située à Limoilou, quartier populaire de Québec. Événement dramatique par excellence la greve met aux prises deux camps principaux: les patrons, propriétaires de l'entreprise, et les travailleurs regroupés dans un syndicat. 
Entre les deux camps, quelques individus au statut problématique et non enviable: les contremaitres.

Le récıt cependant ne privilégıe pas l'affrontement patronal-syndical. Raconté par un narrateur de formation intellectuelle, ouvrier de fraiche date. il est centré essentiellement sur l'univers des grévistes dont il illustre à la fois la solidarité et les divisions. C'est que du côté des ouvriers il y a d'une part les syndicalistes «corrects * comme Paul-Henri qui sympathise mème avec des militants marxistes-léninistes pour qui la grève est l'occasion d'une intense agitation-propagande, comme Charlie, travailleur acharné que les «mous * du syndicat déçoivent et qui plus souvent qu’à son tour, est « en maudit après le syndicat qui couche avec les boss * (p. 59) et d'autre part les « labor-boss* (pour reprendre une expression souvent utilisèe naguère par le groupe marxisteléniniste En lutte!) tels Bertrand Légaré, un permanent syndical représenté comme un «rat" (p. 45) et le président de l'Union, Labrie, décrit comme un collaborateur des patrons.

Comme on peut rapidement le prévoir, étant donné le point de vue privilégié par le narrateur, les syndicalistes « corrects» se lient avec les militants marxistes-léninistes qui leur expliquent comme de bien entendu que si Staline $\propto$ a fait des erreurs», son «point de vue général» était «correct» (p. 78) et se laissent convaincre d'organiser une manifestation à Toronto pour témoigner leur solidarité avec un syndicat frère du Canada anglais pendant que certains trouvent dans le conflit une occasion de régler leurs problèmes personnels (la «militance aidant par exemple à guérir Gérard de son alcoolisme l) et que le narrateur, pour sa part, se défait peu à peu de ses a vieux vêtements a à a l'école de la lutte des classes* (p. 88).

En somme la grève a été une rude épreuve mais l'essentiel a été atteint: "on n'est pius les mêmes» (p. 142) et la roue de l'histoire retourne dans le bon sens: celui du changement. Et comme si ce "message " n'était pas assez clair, le narrateur intervient explicitement à quelques reprises pour dénoncer une civilisation qu'il qualifie «d'hypocrite» (p. 106).

De plus d'une manière, ce premier roman se présente donc comme un catalogue d'images d'Epinal et en cela prête facilement le flanc au mot de Gide: ce n'est pas avec de bons sentiments qu'on fait de la bonne littérature. En quoi réside alors son intérêt? D'abord en ce qu'il évoque - ce qui n'est pas courant dans notre littérature - les problèmes et les luttes de la classe ouvrière et des milieux populaires. Ensuite en ce qu'il pose - mal à mon avis - un problème important: celui du rapport de la littérature au réel, celui de la fonction de celle-ci quand on la conçoit autrement que comme simple divertissement, comme pratique militante: comment, à quelles conditions, une production romanesque peut-elle porter une orientation politique progressiste sans perdre sa spécificité et sa valeur en tant qu'cuvre d'art?

C'est cette problématique qui inspire également le deuxième roman de Gilles Raymond, Un moulin, un village, un pays ${ }^{2}$, publié l'an dernier. Le titre. cette fors, est moins explicite mais le soi-disant extrait de convention collective placé en exergue insistant sur le «droit de gérance» des entreprises annonce 
clairement les couleurs: l'ceuvre à nouveau en sera une de combat. cette fois contre les élites traditionnelies et nouvelles d'une petite ville, Donnacona, possédée par une entreprise tentaculaire, la Domtar.

L'histoire de Donnacona des annéés 1940 jusqu'à aujourd hui constitue la structure porteuse du récit qui comprend trois parties: "L'enfance d'un boss" centrée sur les premières années de celui qu'on peut présumer devoir être le héros du roman, "A qui appartient cette terre ?" organisée autour des rapports de force qui opposent durant les années 1950-1960 élites traditionnelles (incarnées par Piton Paradis, un marchand général) et nouvelles (représentées par Léon Noël, un spéculateur) et enfin "Les élections» mettant aux prıses dans les années 1970 les libéraux de Bourassa et les nationalistes du parti National Souverain (le P.Q. for sure!) unis dans leur opposition de surface contre le peuple qui, a la fin du roman réclame en scandant “Pouvoir populaire! Pouvoir ouvrier!» (p. 203).

La première partie du récit est prometteuse: prise en charge par un narrateur adulte qui se souvient des moments significatifs de sa jeunesse, elle est dominée par la figure de Jean G., enfant qui prend tôt conscience des réalités sociales et économiques - de la domination et de l'oppression vécues par les travailleurs - et qui, au début de l'âge adulte, se voit confronté a a l'alternative suivante: étudier pour s'intégrer à la classe dominante ou pour servir le peuple; Jean G. adopte la première voie tandis que «le Pouette $\%$, son double, préfère "vivre de sa pauvreté d'intellectuel mécontent mais content de sa révolten (p. 85). Três tôt donc l'unité qui caractérise le début du récit est battue en brèche: Jean $G$. de narrateur-héros devient un personnage décrit à la troisième personne et le roman glisse de la fiction autobiographique à une chronique éclatée, partant en diverses directions et qui n'a plus d'autre unité que le schéma idéologique qui lui sert de cadre: Jean $G$. devient un personnage parmi d'autres, vidé de sa substance humaine au profit de son rôle de représentant de la classe dominante.

On peut en dire autant des autres personnages, pantins réduits à une fonction d'illustration du propos de l'auteur: Jos Cantin, l'ouvrier modèle bien que résigné des années 1940-1950. Coco, son fils, incarnant la combativité des travailleurs des années. 1970, Léon Noël, spéculateur et maire de Donnacona, porte-parole des élites traditionnelles. Bernarlandri, son fils * naturel on quelque sorte, symbolisant la nouvelle petite-bourgeoisie nationaliste, Charlie Mingus, I'Indien, premier occupant des lieux dépossédé de sa terre etc. Tous ces personnages sont décrits en termes extrêmement schématiques, comme des figures, des rôles occupant des places bien précises dans le système social mais à peu près jamais dans leur complexité d'êtres vivants.

D'où l'allure sévère, très * roman à thèsen du récit que Raymond essaie pourtant d'alléger par divers moyens: la multiplication des chapitres - pour donner du mouvement à l'écriture - et l'utilisation pour les coiffer de titres qui se veulent humoristiques, la mise en scène ironique mais très appuyée de personnages d'actualité - entre Barnarlandri, Pedalo pour Peladeau, Jo Rond pour Joron etc. - le recours à des résumés didactiques, l'insertion de refrains populaires etc. Mais tout cela, il faut bien le dire, tombe a plat et ne réussit 
pas à faire oublier le caractère pesamment didactique de l'entreprise. D'une certaine manière ce second roman s'avère en retrait par rapport au premier dont les qualités d'écrıture - nerveuse, rapıde, allant drait à l'essentiel, procédant par courts tableaux à la manière impressionnante, pointilliste étaient indéniables: il ne reste plus grand chose de cela dans Un moulin...

Faut-il en conclure pour autant que l'cuure est un échec? D'un point de vue étroitement «littéraire», sans doute. Mais ce n'est pas cela qui intéresse l'auteur, le projet littéraire étant chez lui subordonné au projet politique. Et on sait que la publication du Moulin lui a fourni l'occasion de rencontrer des militants de syndicats et de groupes populaires; ceux-ci ont-ils été réceptifs à son message? Si c'était le cas, on ne pourrait nier que ce roman a produit des effets et une analyse politique sérieuse ne saurait les ignorer. Ce ne serait pas la première fois de toute manière qu'une ceuvre d'une qualité problématıque sur le plan formel joue un rôle politiquement progressiste songeons aux derniers romans d'un Zola par exemple. Par les questions qu'il soulève, ce second roman de Raymond, comme le premier, vaut d'ètre lu surtout si, comme je l'espère, il annonce - maladroitement - un «cours nouveau» dans notre littérature, le surgissement de préoccupations sociales qui lui ont fait cruellement défaut durant la dernière décenniẹ.

Le côté «maitresse d'école» qui agace chez Raymond, on ne le retrouve houreusement pas dans les romans d'Yvon Paré, la Mort d"Alexandre ${ }^{3}$ et de Pierre Filion, Juré craché 4 dont la dimension critique, pour n'être pas tapageuse, est néanmoins indéniable.

Paré, dans la Mort d'Alexandre, raconte quelques journées d'un agars de bois *. Emile Parent, qui gagne - et perd - sa vie dans les forêts et les hôtels de l'Abitibi. Car si, isolés dans les chantiers, on travaille fort durant les mois d'hiver, le printemps venu on descend dans les villes et on se lance à corps perdu dans d'immenses beuveries - les brosses s'étalent parfois sur des semaines et seul le manque d'argent $y$ met fin - et des orgies monstres avec des dames de petite vertu. Puis on rentre aux chantiers pour se refaire du fric et de la santé. Ainsi va la vie, quelque peu monotone et répétitive, entre le cycle du travail et celui de l'alcool.

Ce qui va la perturber dans le cas d'Émile Parent, c'est la mort du père, Alexandre - seul événement véritable du récit et élément qui en assure l'unité - qui, d'une part, va déclencher chez le héros une quête du père, figure quelque peu mythique: homme comme il ne s'en fait plus, un vrai de vrai qui portait les culottes chez lui contrairement à un Théophile Plouffe et, d'autre part, va lui fournir l'occasion d'une prise de contact avec sa famille qu'il a quittée il y a une quinzaine d'années parce qu'il s'y sentait étranger; ses jeunes frères sont aux études et, lui le non-instruit pour reprendre la célèbre formule de Jean Lesage. ne peut que constater qu'ils appartiennent à « un monde où lui n'a pas sa place » (p. 131). Alexandre disparu, Parent n'a plus qu'à rentrer en Abitibi où l'attend la communauté chaleureuse de ses amis.

Ce que Paré évoque surtout dans son roman, c'est la vie de ces gars de chantiers avec ses grandeurs et ses misères. Il montre bien que sı on 
se donne du bon temps avec l'alcool et les femmes, on le paie, non seulement en argent mais en solitude: Parent, pour un, ne peut s'empècher de penser parfois qu'il a peut-ètre manqué sa viè en renonçant à Johanne, une amie de jeunesse. Il sera de mème bouleversé par la mort d'un camarade. Ferron, crevant de ses excès de goinfrerie lors d'une monumentale beuverie. L'amitié elle-même, valeur suprême de cette communauté de mâles, n'est pas toujours dénuée d'arrières-pensées: si Blais. l'hôtelier de Morinville, aime bien Parent et ses amis, if n'oublie jamais ce qu'il gagne a ces relations qui font tourner son commerce. Et les fêtes elles-mèmes tournent aussi à l'occasion à la tristesse sinon à la tragédie; dans un moment de lucidité. Parent dira à la fin du roman : an é rien qu'bon à s'paqu'ter a fraise pis à crier plus fort que tout l'monde pour montrer qu'on a pas peur, qu'on é dé toffes... Rien qu'bons à péter d'la broue tabarnak! On é dé krisses de gros trimpes sales! Dé tabarnak de trimpes... (pp. 210-222).

La description que Paré présente de l'univers des bûcherons, on le voit, comporte une dimension critique, exempte cependant de lourdes démonstrations et de prêches assommants: on est loin du roman à thèse et de ses simplifications. L'écriture, ici, ne perd jamais ses droits. Son mouvement est rapide: phrases nerveuses, paragraphes courts, descriptions précises, réduites a l'essentiel qui ne ralentissent jamais la marche du récit, dialogues nombreux et généralement fort juteux, rendant remarquablement bien l'accent des habitants du Lac St-Jean. Ce qui fait l'intérêt de ce roman, c'est ainsi tout a la fois son propos - faire connaître une réalité humaine quion ne rencontre guère dans notre littérature - et la qualité de l'écriture qui le supporte.

On peut en dire autant - et plus - de l'excellent roman de Pierre Filion auquel la critique, si je me souviens bien, n'a pas accordé toute l'attention qu'il mérite.

Au centre du récit: la révolte d'un père. Alphonse Godon - un colon de Saint-Agricole, hameau au nord de Sainte-Agathe - a la mort de son fils unique tué en 1919 par la grippe espagnole. Révolte provoquée pour une part par cette mort absurbe et affreuse qui signifie la fin de la lignée, les autres enfants étant des filles, et surtout par l'attitude du curé qui réfuse la sépulture chrétienne à Jean-Baptiste dont les parents, vivant en concubinage, sont exclus depuis vingt ans des rangs de l'Église. Ulcéré, le père décide donc d'assurer lui-même l'enterrement de son fils non sans avoir auparavant parcouru à pied la campagne de Sainte-Agathe et le village lui-même, trainant sur son dos la dépouille de son fils et criant sa colère à tous les habitants rencontrés durant son périple, et au premier chef au curé qu'il vient engueuler sur le perron de l'Église.

Cet épisode hautement dramatique sert de fil conducteur au récit qui est pris en charge pour l'essentiel par un témoin de l'événement - alors enfant de onze ans - qui le fait revivre, adulte. sur le mode du souvenir. Cependant ce narrateur n'est pas unique, le roman étant aussi raconté à l'occasion par un narrateur - ou à la troisième personne et par les principaux personnages 
(Alphonse Godon, sa femme Anastasie, son fils) dont on nous fait entendre a quelques reprises la parole. Cette orchestration extrèmement habile des voix narratives est greffée sur une construction d'ensemble rigoureuse et ingénieuse. Filion faisant alterner les chapitres consacrés à l'anecote principale - l'odyssée du père - avec d'autres évoquant le passé des personnages jusqu'au chapitre final qui nous reporte à l'époque immédiatement contemporaine, le narrateur - enfant du début devenu vieil homme tirant les leçons de l'exérience de la colonisation. Cette organisation formelle trés intéressante est par ailleurs mise au service d'un propos passionnant. A loccasion du drame vécu par Godon, c'est uno histoire concrète de la colonisation des pays d'en haut, telle qu'éprouvée dans leur chair par des personnages hors du commun que reconstitue Filion.

Alphonse Godon en effet, ce "volcan d'énergie et de courage* (p. 112). ce géant, incarne une génération d'hommes fiers, indépendants, travailleurs qui, d'une manière humble, sans éclat, ont vécu une épopée héroïque. A vingt ans, par exemple, Alphonse marie Adèle, sa première femme, avec qui il connaît une existence calme et heureuse a Sainte-Agathe. Puis, Adèle devenue folle, il doit la faire enfermer à St-Jean de Dieu d'où elle lui écrira une lettre poignante qui rappelle un peu « la lettre d'amour * d'Aline Dupire dans les Roses sauvages de Ferron. II s'éprend alors d'Anastası qu'il ne peut marier, son premier mariage demeurant toujours valide aux yeux de l'Église et, exclu d'une communauté dominée par une conception étroite du catholicisme, il décide de partır plus haut, au nord de Sainte-Agathe, en plein bois. d'y bâtir maison et d'établir famille: entreprise difficile, ardue qu'il mène a bien grâce à un travail acharné, à beaucoup de patience et de courage, conscient d'accomplir une mission et d'être d'une certaine façon un * conquérant * (p. 37).

Anastasie, sa compagne de misère et de gloire, constitue de même un remarquable type de femme : brave mais lucide, elle est conscience de vivre une histoire où les humbles comme eux n'auront jamais la place d'honneur: * Nous sommes, dit-elle dans un touchant monologue où elle s'adresse tour à tour au fils mort et à l'époux, dans la marge des livres d'histoire, dans l'espace blanc, on parle pas de nous autres, on en parlera jamais, on pèse si peu * (p. 136). Et. se démarquant de Godon qui refuse obstinément la ville et le progrès. plus sage que son homme, elle est consciente que sa vie aurait pu être différente et peut-être plus stimulante ailleurs, sans sombrer toutefois dans les regrets, les jugeant inutiles car ne changeant rien.

Le fils Jean-Baptiste, lui est partagé entre la fidélité au père et à sa vocation naturelle et l'attrait qu'il ressent pour les ailleurs: la ville, les autres pays. II aurait bien voulu par exemple aller à la guerre. pas pour tuer - c'est un doux - mais pour connaître autre chose que l'univers étroit du Nord. La grippe espagnole le tuera avant qu'il puisse réaliser ses rêves comme elle en a tué des milliers d'autres à l'époque - une époque que Filion fait revivre dans des pages magnifiques au quatrième chapitre du roman.

A travers ces trois destins, c'est toute I'histoire de la colonisation qui est évoquée et discutée. Et le romancier en présente une image réaliste: 
oui, cette entreprise par certains côtés fut admirable - en la vivant. toute une génération a affirmé sa grandeur - mais en mème temps absurde: comme le dit le narrateur - adulte aux dernières pages, le développement agricole du Nord a été une xbonne intention catholique dépourvue de réalisme" (p. 186). Filion rejoint ici Paul Villeneuve qui, dans Johnny Bungalow - roman qui présente beaucoup de traits communs avec celui-ci - dressait le mẻme constat pour l'Abitibi.

Je pourrais parler encore longtemps de ce merveilleux roman qui fait si bien revivre toute une époque sans jamais sombrer dans le didactisme. grâce essentiellement à un heureux usage d'une prose toute pénétrée de lyrisme. Filion a réussi à produire une ceuvre dans laquelle un projet intéressant en soi est rendu avec un très grand bonheur d'expression. Son roman constitue la meilleure preuve qu'un roman social, sinon engagé, peut être également une grande réussite littéraire comme le démontre d'ailleurs toute la tradition du grand roman classique des $\mathrm{XIXe}$ et $\mathrm{XXe}$ siècles, un peu éclipsée depuis une vingtaine d'années par les diverses manifestations du courant formaliste; les romans que j'ai évoqués ici, et d'autres, annoncent peut-être le retour - avec des moyens différents, dans de nouvelles formes - à cette tradition longtemps dominante dans le discours romanesque. Pour ma part, je n'en serais pas fâché.

1. Gilles Raymond, Pour sortir de nos cages, Rimouski. Les gens d'en bas, 1979.

2. Gilles Raymond, Un moulin, un village, un pays. Montréal, VLB éditeur, 1982.

3. Yvon Paré, la Mort d'Alexandre, Montréal, VLB éditeur, 1982.

4. Pierre Filion, Juré craché, Montréal, VLB éditeur, 1981. 